\title{
USO DA PLACA ONDA NO TRATAMENTO DAS FRATURAS DIAFISÁRIAS DO FÊMUR NÃO CONSOLIDADAS
}

\author{
THE WAVE PLATE METHOD IN NON UNION FEMORAL SHAFT FRACTURES TREATMENT
}

\author{
Silvia Regina Nogueira Jorge ${ }^{1}$, Luiz Fernando Cocco ${ }^{2}$, Claudio Kawano ${ }^{2}$, Helio Jorge Alvachian Fernandes ${ }^{3}$, \\ FERNANDO BALDY DOS REIS
}

\begin{abstract}
RESUMO
Estudou-se retrospectivamente 25 pacientes com fratura diafisária do fêmur não consolidadas e que foram tratados com placa onda. A consolidação ocorreu em $96 \%$ dos pacientes num tempo médio de 5,32 meses, variando entre três e sete meses. Vinte e um pacientes (84\%) foram considerados como resultados excelentes e bons nos critérios de avaliação final. O método de tratamento não ocasionou diferença no comprimento dos membros. Não ocorreram desvios rotacionais. $\mathrm{O}$ arco de movimento dos quadris e joelhos não foi acometido, embora em quatro pacientes (16\%) tenha-se encontrado limitação na flexão do joelho, esta era prévia ao tratamento com placa onda. Dois pacientes (8\%) tiveram infecção profunda durante o tratamento com a placa onda, recidiva de processo infeccioso prévio. Houve soltura da placa no nono mês de pós-operatório em um paciente (4\%), embora tenha havido a consolidação óssea. Em outro paciente (4\%) a placa onda quebrou um ano e dois meses após a cirurgia, tendo-se trocado a primeira por outra placa onda e posterior consolidação. Mesmo assim, considerou-se falha do método. A placa onda é uma opção de tratamento das fraturas do fêmur não consolidadas pelas suas propriedades biomecânicas favoráveis à consolidação óssea, conferindo estabilidade sem prejudicar o suprimento sangüíneo, com características de síntese biológica.
\end{abstract}

Descritores: Pseudo-artrose; Placas Ósseas; Fraturas do fêmur.

\section{SUMMARY}

Twenty-five patients with non union femoral diaphyseal fractures treated with the wave plate method were retrospectively studied. Union was achieved in 24 patients (96\%) in an average time of 5.32 months, ranging from three to seven months. We observed excellent and good results in 21 patients (84\%) in the endpoint evaluation. This treatment approach didn't cause leg-length discrepancy. No rotational deviations were seen. The range of motion of hips and knees was not affected, although in four patients (16\%) knee flexion restraint was found, but previously to the wave plate treatment. Two patients (8\%) presented a deep infection during the treatment with the wave plate, recurrent to previous infectious process. Loosing plate occurred in the ninth post-operative in one patient (4\%), although bone union has occurred. In another patient (4\%) the wave plate has broken within 14 months postoperatively, leading to the replacement of the first wave plate to a new one with subsequent union. Nevertheless, method failure was considered. The wave plate is a treatment option for non-united femoral fractures due to its biomechanical properties favoring bone union, providing stability without jeopardizing blood intake, with biological synthesis characteristics.

Keywords: Pseudoarthrosis; Bone Plates; Femoral fractures.

\section{INTRODUÇÃO}

Diversas técnicas operatórias são utilizadas para o tratamento das fraturas diafisárias do fêmur no adulto e apresentam algumas complicações, sendo uma delas a pseudo-artrose, rara na literatura e que desafia o médico ortopedista.

Blatter G et al. ${ }^{(1)}$ descreveram em 1990 um estudo biomecânico da placa onda e sua utilização no tratamento das falhas do material de síntese, retardes de consolidação e pseudo-artroses nas fraturas diafisárias do fêmur, objetivando a consolidação óssea. O método proposto constitui uma nova opção de tratamento, sendo de fácil acesso, baixo custo, ratificando as vantagens biológicas e mecânicas da placa onda quando comparadas à placa reta convencional.

O presente trabalho tem o objetivo de avaliar a utilização da placa onda em 25 fêmures (25 pacientes) com fraturas diafisárias do fêmur não consolidadas e demonstrar a eficácia do método.

\section{MATERIAL E MÉTODO}

No período de julho de 1991 a outubro de 1998 no Hospital São Paulo e em duas outras instituições, 25 pacientes portadores de pseudo-artrose foram tratados com o método da placa em onda sendo utilizadas placas DCP (Dynamic Compression Plate) e LCDCP (Low Contact Dynamic Compression Plate) largas de grandes fragmentos e enxerto bicortical do osso ilíaco, sendo acompanhados prospectivamente.

Foi desenvolvido um protocolo onde os pacientes foram seguidos até o final deste trabalho.

Considerou-se fraturas diafisárias aquelas localizadas abaixo do trocânter menor até o tubérculo dos adutores ou a proeminência dos côndilos ${ }^{(2)}$

Adotou-se a classificação AO - ASIF (Arbeitsgemeinschaft für Osteosynthesefragen - Association for Study of Internal Fixation) ${ }^{(3)}$ para a classificação das fraturas (Figura 1) e para as

Trabalho realizado Escola Paulista de Medicina - Universidade Federal de São Paulo - DOT.EPM/UNIFESP.

Correspondência: Rua Borges Lagoa, 783 - 5o andar - São Paulo - SP - CEP: 04038-32 - Departamento de Ortopedia e Traumatologia - e-mail: heliofernandes@terra.com.br

1. Mestre em Ortopedia

2. Médico Residente do $3^{\circ}$ ano

3. Doutor - Chefe do Setor do Trauma da Disciplina de Traumatologia

4. Prof. Livre Docente da Disciplina de Traumatologia 
pseudo-artroses a classificação preconizada por Weber( ${ }^{(4)}$ (Figura 2).

\section{Critérios de inclusão:}

Foram incluídos pacientes que tiveram fraturas diafisárias do fêmur tratadas com placas de compressão, e que evoluíram com retarde de consolidação, pseudo-artrose ou falha do material de síntese (quebra ou soltura). Indicou-se a placa onda quando eram encontrados sinais radiográficos de instabilidade no foco da fratura: identificação do osso avascular, persistência de movimento no foco com formação de calo irritativo, absorção óssea ou falha do material de síntese, como quebra, soltura ou deformidade plástica.

\section{Critérios de exclusão:}

Foram excluídos pacientes que apresentaram infecção e aqueles tratados com haste intramedular.

Coletou-se dados dos pacientes, segundo número de ordem, iniciais do nome, hospital de origem, idade, sexo, lado acometido, localização anatômica, classificação da $\mathrm{AO}$, data da cirurgia da placa onda, indicação da placa onda e distúrbios da consolidação (Tabela 1).

Dos 25 pacientes operados, 14 eram do lado direito (56\%) e 11 do esquerdo (44\%), 21 eram fechadas (84\%) e quatro expostas (16\%) na fase aguda. A idades dos pacientes variou entre 16 e 53 anos, com média de 26,68 anos, sendo 19 masculinos (76\%) e seis femininos (24\%)

Quanto à localização da fratura, um paciente $(4 \%)$ apresentou fratura no terço proximal, 22 (88\%) no terço médio e dois (8\%) no terço distal do fêmur acometido. As fraturas foram classificadas de acordo com a classificação $A O$, sendo quatro do tipo $\mathrm{A} 2(16 \%)$, duas do tipo $\mathrm{A} 3(8 \%), 12$ do tipo B1 (48\%), uma do tipo B2 (4\%), duas do tipo B3 (8\%) e quatro do tipo C1 (16\%). Observou-se a falta de apoio na cortical medial nas fraturas diafisárias do fêmur em 23 pacientes (92\%).

Foi utilizado enxerto ósseo esponjoso na cirurgia inicial de estabilização da fratura com placa e parafusos em 12 pacientes (48\%).

Três pacientes (12\%) foram submetidos a duas cirurgias antes da indicação da placa onda e o restante, 22 (88\%), apenas uma cirurgia pregressa. Todas as cirurgias iniciais foram com placas retas DCP e nos pacientes com duas intervenções cirúrgicas anteriores, a placa reta havia sido substituída por outra placa reta. Um paciente foi tratado com a placa DCP, mas com síntese biológica (placa ponte) e a segunda cirurgia foi realizada para colocação de enxerto ósseo; mesmo assim houve falência do material de síntese.

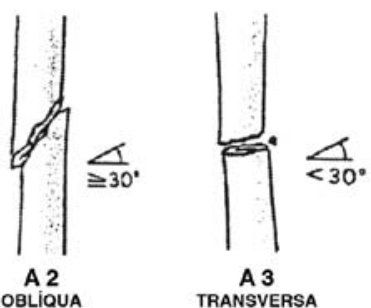

Optou-se pela troca da placa em 16 pacientes por quebra da síntese (64\%), três por ausência da consolidação (12\%) e seis, por soltura do material (24\%).

Foram identificados 12 pacientes com fratura não consolidada do fêmur (48\%) e retarde de consolidação em 13 pacientes (52\%) e segundo a classificação( ${ }^{(30)}$ para pseudo-artroses, 10 pacientes $(77 \%)$ com pseudo-artrose hipertrófica e três (23\%) com pseudo-artrose atrófica.

\section{DESCRIÇÃO DA TÉCNICA CIRÚRGICA:}

O paciente é posicionado em decúbito dorsal em mesa cirúrgica comum com discreta elevação do quadril do lado a ser operado (coxim).

Realiza-se uma incisão na face lateral da coxa, tendo como parâmetros o trocânter maior e o côndilo femoral lateral, procurando respeitar a mesma incisão utilizada na primeira cirurgia. A fáscia lata é abordada através de uma incisão paralela à realizada na pele, e o acesso muscular realizado de acordo com a cirurgia pregressa (trans ou retro-vasto lateral). Abordado

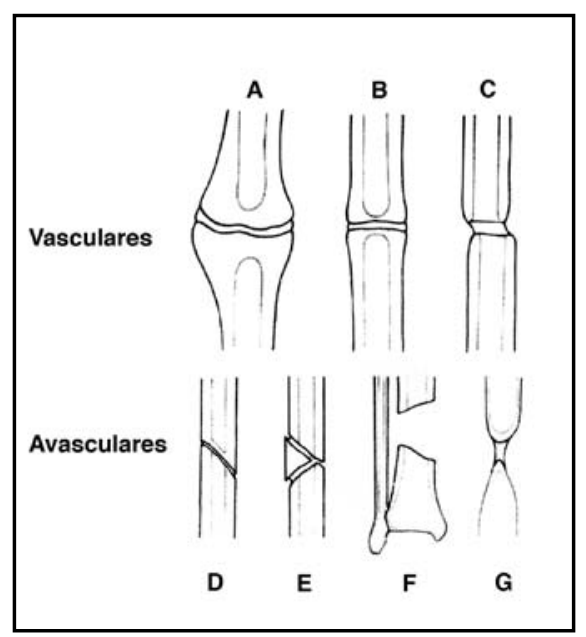

Vasculares: $A=$ hipertrófica (pata de elefante), $B=$ normotrófica (pata de cavalo)

$\mathrm{C}=$ hipotrófica. Avasculares: $\mathrm{D}=$ cunha de torção, $\mathrm{E}=$ multifragmentada

$\mathrm{F}=$ falha óssea, $\mathrm{G}=$ atrófic

Figura 2 - Classificação de Weber para pseudo-artroses. o plano ósseo, são descoladas as aderências e osteotomizadas as eventuais reações periostais para que se possa retirar o material de síntese inicial posicionado lateralmente ao osso.

Após identificação do foco da pseudoartrose, corrigem-se eventuais desvios e procura-se desperiostizar o mínimo possível.

Faz-se a modelagem da placa em forma de onda na sua porção média, onde a extensão varia de acordo com as dimensões da pseudo-artrose, entretanto, com o menor comprimento possível (Figura 3). Retira-se enxerto bicortical do osso ilíaco, contra lateral ao lado operado, com a espessura da onda previamente moldada (um centímetro em média). Nos casos onde já houvessem sido abordada esta região em cirurgia anterior, o novo enxerto era retirado do lado ipsilateral ao fêmur acometido.

A fixação da placa DCP ou LCDCP larga moldada ao osso é feita através de parafusos de grandes fragmentos proximal e distalmente (oito a 10 corticais proximais e distais), modificando o posicionamento da placa para não coincidir os orifícios dos parafusos anteriores. Quando a área de enxerto é considerada muito grande ou instável, pode-se fixá-lo à diáfise do fêmur com parafusos, transfixando-o através da placa, sem a necessidade da rosca chegar na cortical medial. 
Após a fixação da placa e enxerto, procede-se a lavagem da ferida operatória com aproximadamente cinco litros de soro fisiológico para remoção de coágulos e tecidos desvitalizados. A seguir, fazse a sutura por planos, deixando dois drenos de aspiração. Estes devem ser retirados entre 24 a 48 horas após a cirurgia, dependendo do volume de drenagem.

Os pacientes são mantidos com curativo oclusivo e o joelho fletido a $90^{\circ}$ por três dias. Movimentos ativos foram estimulados desde o primeiro pósoperatório. Intensifica-se a fisioterapia com exercí-

\begin{tabular}{|c|c|c|c|c|c|c|c|c|c|}
\hline $\begin{array}{c}\text { Numero } \\
\text { de } \\
\text { Ordem }\end{array}$ & $\begin{array}{c}\text { Hospital } \\
\text { de } \\
\text { Origem }\end{array}$ & Idade & Sexo & $\begin{array}{c}\text { Lado } \\
\text { Acometido }\end{array}$ & $\begin{array}{l}\text { Localizaçăo } \\
\text { da Fratura }\end{array}$ & $\begin{array}{c}\text { Classificação } \\
\text { da Fratura } \\
\text { Inicial (AO) }\end{array}$ & $\begin{array}{l}\text { Data da } \\
\text { Placa } \\
\text { Onda }\end{array}$ & Indicaçăo & $\begin{array}{l}\text { Distúrbio de } \\
\text { Consolidaçăo }\end{array}$ \\
\hline 1 & HSP & 18 & M & $E$ & 1/3 MÉDIO & B3 & JUL91 & QUEBRA & PSEUDOARTROSE H \\
\hline 2 & HSP & 21 & $F$ & $E$ & 1/3 MÉDIO & B1 & JUL91 & QUEBRA & RETARDE \\
\hline 3 & HSP & 37 & $\mathrm{~F}$ & $\mathrm{E}$ & 1/3 MÉDIO & B2 & MAR/92 & QUEBRA & PSEUDOARTROSE A \\
\hline 4 & HSP & 53 & $\mathrm{M}$ & $D$ & 1/3 MÉDIO & C1 & SET/92 & QUEBRA & RETARDE \\
\hline 5 & HSP & 28 & M & D & 1/3 MÉDIO & A2 & NOV/92 & $\begin{array}{l}\text { SEM } \\
\text { CALO }\end{array}$ & RETARDE \\
\hline 6 & HSP & 22 & M & D & 1/3 MÉDIO & B1 & DEZ/92 & $\begin{array}{l}\text { SEM } \\
\text { CALO }\end{array}$ & PSEUDOARTROSE H \\
\hline 7 & HSP & 29 & M & D & 1/3 MEDIO & B3 & ABR/93 & QUEBRA & RETARDE \\
\hline 8 & HSP & 27 & M & $\mathrm{D}$ & 1/3 MÉDIO & B1 & AGO/94 & QUEBRA & RETARDE \\
\hline 9 & HSP & 51 & $\mathrm{~F}$ & $\mathrm{D}$ & 1/3 MÉDIO & A2 & OUT/94 & SOLTURA & RETARDE \\
\hline 10 & HSP & 21 & $\mathrm{~F}$ & $\mathrm{E}$ & 1/3 MÉDIO & A2 & MAR/95 & QUEBRA & PSEUDOARTROSE H \\
\hline 11 & HSP & 25 & M & D & 1/3 MÉDIO & $\mathrm{B} 1$ & ABR/96 & QUEBRA & RETARDE \\
\hline 12 & HSP & 23 & M & $\mathrm{E}$ & 1/3 MÉDIO & B1 & MAL/ 96 & SOLTURA & RETARDE \\
\hline 13 & HSP & 20 & M & D & $\begin{array}{c}1 / 3 \\
\text { PROXIMAL } \\
\end{array}$ & B1 & OUT/97 & QUEBRA & RETARDE \\
\hline 14 & HSP & 29 & M & $\mathrm{D}$ & 1/3 DISTAL & B1 & OUT/07 & QUEBRA & PSEUDOARTROSE H \\
\hline 15 & HSP & 17 & $\mathrm{~F}$ & $D$ & 1/3 MÉDIO & $\mathrm{B} 1$ & OUT/97 & \begin{tabular}{|l} 
QUEBRA \\
\end{tabular} & PSEUDOARTROSE A \\
\hline 16 & HSP & 30 & M & $\mathrm{D}$ & 1/3 MÉDIO & C1 & NOV/97 & SOLTURA & PSEUDOARTROSE H \\
\hline 17 & HSP & 27 & M & $\mathrm{E}$ & 1/3 MÉDIO & A2 & MAR/98 & SOLTURA & RETARDE \\
\hline 18 & HSP & 26 & M & $\mathrm{E}$ & 1/3 MÉDIO & B1 & MAR/98 & SOLTURA & PSEUDOARTROSE H \\
\hline 19 & HSP & 24 & M & D & 1/3 MÉDIO & $\mathrm{B} 1$ & ABR/98 & SOLTURA & RETARDE \\
\hline 20 & HSP & 16 & $\mathrm{~F}$ & $D$ & 1/3 MÉDIO & B1 & MAl/98 & QUEBRA & PSEUDOARTROSE H \\
\hline 21 & HSP & 21 & M & $\mathrm{E}$ & 1/3 MÉDIO & A3 & MAl/98 & $\begin{array}{l}\text { SEM } \\
\text { CALO } \\
\end{array}$ & PSEUDOARTROSE A \\
\hline 22 & HSP & 16 & M & $D$ & 1/3 MÉDIO & B1 & JUL98 & QUEBRA & RETARDE \\
\hline 23 & HSP & 19 & M & $\mathrm{E}$ & 1/3 DISTAL & $\mathrm{A} 3$ & JUL98 & QUEBRA & PSEUDOARTROSE H \\
\hline 24 & Limeira & 46 & M & $\mathrm{E}$ & 1/3 MEDIO & C1 & SET/98 & QUEBRA & PSEUDOARTROSE H \\
\hline 25 & Diadema & 41 & M & $\mathrm{E}$ & 1/3 MÉDIO & C1 & OUT/98 & QUEBRA & PSEUDOARTROSE H \\
\hline
\end{tabular}

Abreviações: $\mathrm{HSP}=$ Hospital São Paulo; $M=$ masculino; $F=$ feminino; $E=$ esquerdo; $D=$ direito; $P=$ placa; $\mathrm{H}=$ hipertrófica; $\mathrm{A}=$ atrófica.

Fonte: Hospital São Paulo, Hospital de Limeira e Hospital Estadual de Diadema

Tabela 1 - Dados dos 25 pacientes com fratura diafisária do fêmur não consolidada tratados com placa onda segundo número de ordem, hospital de origem, iniciais do nome, idade, sexo, lado acometido, localização anatômica, classificação $A O$, data da cirurgia da placa onda, indicação da placa onda e alteração do processo de consolidação.
A partir desta pontuação avaliou-se os resultados do tratamento com placa onda. Considerou-se EXCELENTE os pacientes que atingiram 15 pontos, BOM os que ficaram entre 14 e 10 pontos, REGULAR aqueles entre nove e sete pontos e MAU os pacientes classificados com menos de sete pontos (Tabela 2).

\section{RESULTADOS}

Os resultados do tratamento com placa onda dos 25 pacientes foram avaliados no período pós-operatório. Os dados segundo número de ordem, data da revisão final tempo de consolidação, complicações durante o tratamento, discrepância de membros inferiores, desvios angulares $e$ mobilidade articular do joelho após consolidação encontram-se na Tabela 3.

ativos assistidos

após a retirada do dreno.

A carga inicial no membro operado foi de $20 \%$ do peso corporal. A descarga total é permitida quando sinais radiográficos demonstrando consolidação óssea foram evidentes.

Foram considerados retarde de consolidação a não consolidação óssea em até seis meses após a cirurgia inicial com placa reta, e pseudo-artrose, as fraturas não consolidadas com mais de seis meses.

\section{Critérios de avaliação:}

Encurtamento: equalização dos membros inferiores nota cinco, com encurtamento pré-existente à utilização da placa onda receberam nota três e os com encurtamento maior do que existia previamente à cirurgia com a placa onda, nota zero.

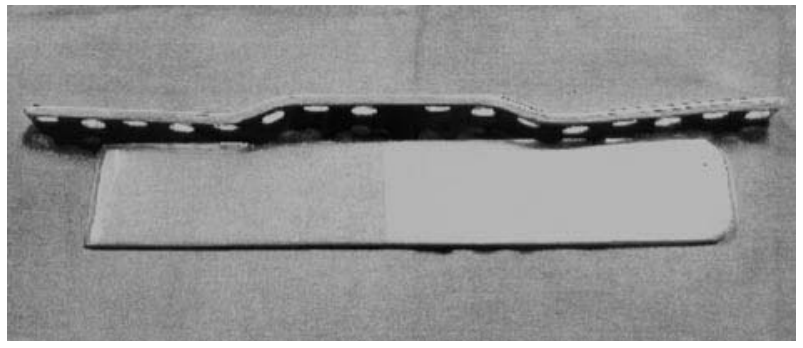

Figura 3 - Placa moldada com as duas extremidades elevadas aproximadamente $0,5 \mathrm{~cm}$ de uma superfície reta.
A consolidação óssea ocorreu em $96 \%$ dos pacientes, num tempo médio de 5,32 meses, variando entre três e sete meses. O tempo de seguimento dos pacientes variou entre cinco meses e dois anos e nove meses.

Não houve alterações no comprimento final dos membros inferiores em 18 pacientes $(72 \%)$.

Em 20 pacientes (80\%) não se observou desvios angulares no final do tratamento. Quatro (16\%) obtiveram a consolidação do fêmur com desvio em varo e um (4\%) consolidou com desvio em valgo. Não foram constatados desvios rotacionais.

$\mathrm{O}$ arco de movimento da articulação do quadril não foi alterado em nenhum caso. A articulação do joelho não se apresentou restrita em $84 \%$ dos casos

Tabela 2 - Pontuação avaliando encurtamento, desvios angulares e mobilidade articular.
Desvios angulares (varo e valgo): sem desvio nota cinco, com desvio até cinco graus nota três, entre cinco e 10 graus nota um, e nota zero para os quais apresentaram desvios superiores a 10 graus.

Mobilidade do joelho: arco de movimento normal nota cinco, flexão de até 90 graus nota três e os com flexão menor que 90 graus nota zero.
(21 pacientes). Houve limitação da flexão em 12\% dos casos. Um paciente (4\%) apresentou o joelho rígido antes da indicação da placa onda. Durante a cirurgia, após manipulação, conseguiuse um arco de movimento de cinco a 20 .

As complicações encontradas foram: um paciente com fratura, um com soltura da placa, um com quebra da placa e dois com infecção. 
A avaliação clínica dos 25 pacientes se encontra na Tabela 4.

\section{DISCUSSÃO}

O processo de consolidação das fraturas envolve contato ósseo entre os fragmentos, estabilidade e suprimento sanguíneo adequado para que não seja prejudi-

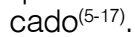

A interrupção no processo de consolidação óssea ocasiona o retarde de consolidação ou a pseudo-artrose. Alguns autores baseiam-se no tempo de evolução e consideram retarde de consolidação a não consolidação óssea em até seis meses, variando entre quatro a seis meses, e pseudo-artrose

\begin{tabular}{|c|c|c|c|c|c|c|c|}
\hline $\begin{array}{l}\text { NúMERO } \\
\text { DE } \\
\text { ORDEEM }\end{array}$ & $\begin{array}{l}\text { REVISÃo } \\
\text { FINAL }\end{array}$ & $\begin{array}{l}\text { TEMPO DE } \\
\text { CONSOLIDAĢĀO }\end{array}$ & COMPLICAÇŌES & ENCURTAMENTO & $\begin{array}{l}\text { DESVIOS } \\
\text { ANGULARES }\end{array}$ & $\begin{array}{l}\text { MOBILIDADE } \\
\text { ARTICULAR }\end{array}$ & $\begin{array}{c}\text { RESULTADO } \\
\text { FINAL }\end{array}$ \\
\hline 1 & FEV/92 & 7 & NÅO HOUVE & NÃO & NĂO & NORMAL & EXCELENTE \\
\hline 2 & NOV/91 & 5 & NĀO HOUVE & NĀOO & NĀO & NORMAL & EXCELENTE \\
\hline 3 & ABR/95 & 6 & NÅO HOUVE & NÅOO & NÁO & NORMAL & EXCELENTE \\
\hline 4 & ABR/93 & 6 & NÃO HOUVE & NĀO & VARO $=11^{\circ}$ & NORMAL & $\mathrm{BOM}$ \\
\hline 5 & MAR/94 & 6 & NÃO HOUVE & NĀO & NĀO & NORMAL & EXCELENTE \\
\hline 6 & FEV/94 & 3 & NÅO HOUVE & NĀO & NĀO & NORMAL & EXCELENTE \\
\hline 7 & FEV/95 & 7 & $\begin{array}{l}\text { FRAT. SUPRA } \\
\text { COND. FÊMUR } \\
\end{array}$ & $05 \mathrm{~cm}$ & VARO $=10^{\circ}$ & FLEXÃO $=90^{\circ}$ & REGULAR \\
\hline 8 & JUN/96 & 5 & NÃO HOUVE & NĀO & NÃO & NORMAL & EXCELENTE \\
\hline 9 & JUU 97 & 6 & NÃO HOUVE & NĀO & VARO $=18^{\circ}$ & NORMAL & $\mathrm{BOM}$ \\
\hline 10 & OUT/95 & 5 & NÃO HOUVE & NÅO & VALGO $=03^{\circ}$ & NORMAL & BOM \\
\hline 11 & OUT/96 & 5 & NĀO HOUVE & NĀO & NĀO & NORMAL & EXCELLENTE \\
\hline 12 & MAR/97 & 4 & NAOO HOUVE & $02 \mathrm{CM}$ & VARO $=18^{\circ}$ & NORMAL & REGULAR \\
\hline 13 & MAl/99 & 6 & $\begin{array}{l}\text { QUEBRA DA } \\
\text { PLACA ONDA }\end{array}$ & NĀO & NÃO & NORMAL & MAU \\
\hline 14 & $\mathrm{SET} / 98$ & 7 & NÃO HOUVE & NĀO & NĀO & NORMAL & EXCELENTE \\
\hline 15 & JUL98 & 4 & NÃO HOUVE & $2,5 \mathrm{~cm}$ & NĀO & FLEXĀO $=110^{\circ}$ & BOM \\
\hline 16 & MAR/99 & 7 & $\begin{array}{l}\text { RECIDIVA DE } \\
\text { INFECÇĀOO }\end{array}$ & $02 \mathrm{~cm}$ & NĀO & FLEXĀO $=20^{\circ}$ & REGULAR \\
\hline 17 & JAN/99 & 6 & NAOO HOUVE & NĀO & NĀO & NORMAL & EXCELENTE \\
\hline 18 & JAN/99 & 5 & NĀO HOUVE & NĀOO & NĀO & NORMAL & EXCELENTE \\
\hline 19 & JAN/99 & 3 & NÃO HOUVE & NĀO & NĀO & NORMAL & EXCELENTE \\
\hline 20 & ABR/99 & 6 & $\begin{array}{l}\text { RECIDIVA DE } \\
\text { INFECÇĀO }\end{array}$ & NĀO & NĀO & NORMAL & EXCELENTE \\
\hline 21 & MAI/99 & 7 & $\begin{array}{l}\text { SOLTURA DA } \\
\text { PLACA ONDA }\end{array}$ & $02 \mathrm{~cm}$ & NĀO & FLEXĀO $=120^{\circ}$ & BOM \\
\hline 22 & FEV/99 & 5 & NÃO HOUVE & NÃO & NÃO & NORMAL & EXCELENTE \\
\hline 23 & ABR/99 & 4 & NĂO HOUVE & NÃO & NĂO & NORMAL & EXCELENTE \\
\hline 24 & ABR/99 & 5 & NÃO HOUVE & $02 \mathrm{CM}$ & NĀO & NORMAL & $\mathrm{BOM}$ \\
\hline 25 & ABR/99 & 3 & NĀO HOUVE & $02 \mathrm{CM}$ & NĀOO & NORMAL & BOM \\
\hline
\end{tabular}

Fonte: Hospital São Paulo, Hospital de Limeira e Hospital Estadual de Diadema

Tabela 3 - Resultado final do tratamento de 25 pacientes com fratura diafisária do fêmur não consolidada tratada com placa onda, segundo data da revisão final, tempo de consolidação, complicações, encurtamentos, desvios angulares e mobilidade articular do joelho. rias do fêmur não consolidadas, em razão do uso da placa DCP inicialmente, que deveria ser retirada, mas mesmo assim já estaria provocando uma lesão na circulação periostal devido ao contato ósseo sob a placa. Assim, procurou-se não produzir outra lesão vascular, desta vez medular, pela mudança da técnica inicial, o que ocorreria caso fosse utilizada a haste intramedular.

$\mathrm{O}$ arqueamento do fêmur determina uma força de tração na cortical lateral e compressão na medial, podendo provocar quando não ocorre a consolidação acima de seis meses $^{(3,18-22)}$ Outros acreditam que os limites entre o retarde de consolidação e a pseudo-artrose sejam imprecisos e consideram pseudo-artrose qualquer desvio no processo de consolidação, pois seria a evolução natural de um retarde de consolidação desde que não haja intervenção médica ${ }^{(4-6,12,23,24)}$.

As causas de pseudo-artrose estão relacionadas a: a) gravidade da fratura envolvendo a lesão de partes moles, localização, cominuição, infecção, lesões arteriais e nervosas associadas; b) instabilidade do método de fixação c) redução inadequada; d) falta de contato ósseo $(6-8,10,12,16)$.

O método escolhido para este estudo preconiza a utilização do enxerto ósseo córtico-esponjoso em todos os pacientes, independentemente do tipo de pseudoartrose envolvida, acreditando ser o fator biológico determinante no processo de consolidação.

O tecido fibroso entre os fragmentos ósseos nos vinte e cinco pacientes tratados com a placa onda não foi removido com a finalidade de preservar a vascularização e ainda associou-se o enxerto ósseo cortiço-esponjoso, garantindo a viabilidade deste tecido.

Escolheu-se a placa onda para tratamento das fraturas diafisá-

\begin{tabular}{|c|c|c|c|c|}
\hline $\begin{array}{c}\text { NÚMERO DE } \\
\text { ORDEM }\end{array}$ & ENCURTAMENTO & $\begin{array}{c}\text { DESVIOS } \\
\text { ANGULARES }\end{array}$ & $\begin{array}{c}\text { MOBILIDADE } \\
\text { ARTICULAR }\end{array}$ & $\begin{array}{c}\text { TOTAL DE } \\
\text { PONTOS }\end{array}$ \\
\hline 1 & 5 & 5 & 5 & 15 \\
\hline 2 & 5 & 5 & 5 & 15 \\
\hline 3 & 5 & 5 & 5 & 15 \\
\hline 4 & 5 & 0 & 5 & 10 \\
\hline 5 & 5 & 5 & 5 & 15 \\
\hline 6 & 5 & 5 & 5 & 15 \\
\hline 7 & 3 & 1 & 3 & 7 \\
\hline 8 & 5 & 5 & 5 & 15 \\
\hline 9 & 5 & 0 & 5 & 10 \\
\hline 10 & 5 & 3 & 5 & 13 \\
\hline 11 & 5 & 5 & 5 & 15 \\
\hline 12 & 3 & 0 & 5 & 8 \\
\hline 13 & 0 & 0 & 0 & 0 \\
\hline 14 & 5 & 5 & 5 & 15 \\
\hline 15 & 3 & 5 & 3 & 13 \\
\hline 16 & 3 & 5 & 0 & 8 \\
\hline 17 & 5 & 5 & 5 & 15 \\
\hline 18 & 5 & 5 & 5 & 15 \\
\hline 19 & 5 & 5 & 5 & 15 \\
\hline 20 & 5 & 5 & 5 & 15 \\
\hline 21 & 3 & 5 & 3 & 13 \\
\hline 22 & 5 & 5 & 5 & 15 \\
\hline 23 & 5 & 5 & 5 & 15 \\
\hline 24 & 3 & 5 & 5 & 13 \\
\hline 25 & 3 & 5 & 5 & 13 \\
\hline
\end{tabular}

Fonte: Hospital São Paulo, Hospital de Limeira e Hospital Estadual de Diadema

Tabela 4 - Avaliação clínica dos 25 pacientes com fraturas diafisárias do fêmur não consolidadas tratadas com placa onda, segundo número de ordem, encurtamento, desvios angulares, mobilidade articular e somatória do número de pontos. falha da síntese (fratura por fadiga) em razão da falta de apoio medial (Figura 4). A placa onda altera esta distribuição devido às suas propriedades mecânicas. Sabe-se que a partir da mecânica elementar que quando uma área é submetida a uma carga excêntrica gera-se tensão que determina a força de arqueamento, e se a área for duplicada, esta força será reduzida a um terço (Figura 5).

A placa onda utilizada nas fraturas da diáfise do fêmur sem apoio medial aumenta a área de carga que passa a ser compreendida desde a região do contato medial até a placa onda e distribui meIhor a força de arqueamento, diminuindo a força de compressão na cortical medial e deslocando a força de tração para a placa. Neste caso, a fibra neutra, linha livre da ação de forças na transição entre a região de compressão e tração, desloca-se para o espaço entre o osso e a placa (Figura 6).

Havendo sustentação medial, a placa onda promove um deslocamento da fibra neutra, em direção à placa, de modo que a fratura encontra-se sobre uma distribuição 
de forças seguindo os mesmos princípios biomecânicos descrito acima pelo aumento da área de carga, mantendo a compressão no foco fraturário (Figura 7).

O tempo de consolidação das fraturas diafisárias do fêmur não consolidadas tratadas com placa onda ocorreu em média de 5,32 meses, variando entre três e sete meses, considerando-se um bom resultado quando comparado à literatura pertinente ${ }^{(19,20,25-31)}$.

Wu e Shi(22) utilizaram quatro métodos de tratamento para a pseudo-artrose, a haste intramedular apresentou o menor tempo de consolidação, três meses, e a placa reta quatro meses.

Qualquer método escolhido para tratamento das pseudo-artroses apresenta complicações: encurtamentos, desvios angulares e rotacionais e rigidez articular como as seqüelas mais freqüentes ${ }^{(18,22,32)}$.

Avaliando o comprimento do membro acometido pode-se afirmar que o método eleito não provoca encurtamento, pois todos os pacientes que apresentaram dismetria dos membros (28\%), apresentavam esta alteração previamente a realização da placa onda. Além disso, com a preservação do tecido fibroso interfragmentar e a inexistência de ressecção óssea, não há como o método provocar ou contribuir com a discrepância entre os membros.

A articulação do quadril apresentou arco de movimento normal em todos os pacientes e o joelho não foi comprometido pelo método, pois os pacientes que consolidaram com limitação do arco de movimento, apresentavam alterações prévias.

\section{REFERÊNCIAS BIBLIOGRÁFICAS}

1. Blatter G, Weber BG. Wave plate osteosynthesis as a salvage procedure. Arch Orthop Trauma Surg 1990; 109: 330-3.

2. Johnson KD. Femoral shaft fractures. In: Browner BD, Jupiter J, Levine AM. Skeletal trauma. Philadelphia: Saunders; 1992. p.1525-98.

3. Muller ME, Allgöwer M, Scheider R, Willenegger H. Manual of internal fixation. 2nd ed. Berlin: SpringerVerlag; 1979. p. 333-50.

4. Weber BG, Cech O. Pseudarthrosis. Bern: Hans Huber; 1976.

5. Compere EL. Fracturas: atlas y tratamiento. 5th ed. México: Interamericana; 1964. p. 385

5. Compere EL. Fracturas: atlas y tratamiento. 5th ed. México: Interamericana; 1964. p. 385 .
6. Ficat P, Durroux R, Metton G. Pseudarthrose diaphysaire et consolidation osseuse. Rev Chir Orthop Ficat P, Durroux R, Metton G. Pseudarth

7. Heiple KG, Herdon CH. The pathologic physiology of nonunion. Clin Orthop. 1965; 43: 11-21.

7. Meiple KG, Herdon CH. The pathologic physiology of nonunion. Clin Orthop. 1965; 43: $11-4$

8. Marmor L, Wu D. Repair of a 10-year ununited fracture. Clin Orthop. 1965; 43: 165-76.
9. Maurer P, Evrard J, Van Houtte Mazabraud A. Avec la collaboration de Lemoine A, Zucman J, Cahen

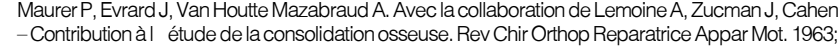
52: 689-702.

10. McKibbin B. Repair of fractures. In: Watson-Jones R. Fractures and joint injuries. 6th ed. Edinburgh: Churchill Livingstone; 1982. p. 14-26.

11. Perren SM. The biomechanics and biology of internal fixation using plates and nails. Orthopedics 1989; 12: 21-34

12. Rand JA, Berquist TH. Fracture healing: Imaging of orthopaedic trauma and surgery. Philadelphia: Saunders; 1986. p. 51-90.

13. Riemer BL, Butterfield SL, Burke CJ, Mathews D. Immediate plate fixation of highly comminuted femoral diaphyseal fractures in blunt polytrauma patients. Orthopedics 1992; 15: 907-16.

14. Riemer BL, Foglesong ME, Miranda MA. Femoral plating. Orthop Clin North Am. 1994; 25: 625-33.

15. TruetaJ. Lavascularisation dês os etI ostéogénèse. Rev Chir Orthop Reparatrice Appar Mot. 1958; 44: 3-23.

16. Trueta J. Nonunion of fractures. Clin Orthop. 1965; 43: 23-35.

17. Trueta J. Ostéoblastes et chondroblastes, relations avec lê millieu intérieur. Rev Chir Orthop Reparatrice Appar Mot. 1966; 52: 711-2
A literatura não relatou quebra do material de síntese ou mesmo soltura da placa(30) pela propriedade da placa onda em distribuir a força cíclica pela extensa área da onda, em vez de se concentrar numa região restrita como ocorre com a placa reta. Testes biomecânicos, entretanto, mostram que a placa onda tende a quebrar no orifício do parafuso distal à curva.

A consolidação óssea ocorre sob a placa devido ao enxerto ósseo, que estimula o processo regenerativo permitindo a penetração dos vasos sangüíneos, e ao excelente sistema mecânico(1) que altera a distribuição de forças, permitindo que a consolidação ocorra sem submeter o material de síntese à sobrecarga.

Embora tenha havido falência da síntese em um dos pacientes e soltura da placa em outro, os resultados foram bons e excelentes em $84 \%$ dos casos, ratificando a placa onda como uma excelente opção de tratamento nos pacientes com fratura do fêmur tratados inicialmente com placa DCP sem sucesso.

\section{CONCLUSÕES}

1.O tratamento com placa onda das fraturas diafisárias do fêmur não consolidadas apresenta bons e excelentes resultados, com alto índice de consolidação (96\%) e amplia as opções de tratamento.

2.A avaliação clínica final dos pacientes mostrou $84 \%$ de excelentes e bons resultados, demonstrando a eficácia do método de tratamento.

3.O material utilizado no tratamento com a placa onda é de baixo custo, sendo disponível na maioria dos serviços em nosso meio.

18. BenoitJ, Cirotteau Y, Huard C, Tomeno B. Étude critique dês échecs dans lêtraitement dês fractures fraîches de la diaphyse fémorale. Rev Chir Orthop Reparatrice Appar Mot. 1974; 60: 464-83.

19. Harper MC. Ununited fractures of the femur stabilized with the flutted rod. Clin Orthop. 1984; 190 273-8.

20. Muller ME, Thomas RJ. Treatment of non-union in fractures of long bones. Clin Orthop.1979; 138: 141-53.

21. Oest O. Pseudarthrose avec et sans fracture d'implant. Acta Orthop Belq. 1971; 37: 726-30.

22. Wu CC, Shih CH. Treatment of 84 cases of femoral nonunion. Acta Orthop Scand. 1992; 63: $57-60$.

23. Cozen L. Spontaneous union of an ununited fracture. Clin Orthop. 1965; 43:161-3.

24. Jones K. Treatment of infected nonunion of the tibia through the posterolateral approach. Clin Orthop. 1965; 43:103-9

25. Boyd HB. Treatment of ununited fractures of the long bonés. J Bone Joint Surg Am. 1965; 47: 167-8.

26. Boyd HB, Anderson LD, Johnston DS. Changing concepts in the treatment of nonunion. Clin Orthop. 1965; 43: 37-53.

27. Crenshaw AH. Delayed union and nonunion of fractures. In: Campbell's operative orthopaedics. 7 th ed. St. Louis: Mosby; 1987. p. 2053-118.

28. Judet R, Patel A. Décortication ostéo-musculaire: révision de 1150 cas. Rev Chir Orthop Reparatrice Appar Mot. 1972; 58: 147-50.

29. Kempf I, Grosse A, RigautP. The treatment of noninfected pseudarthrosis of the fêmur and tibia with locked intramedullary nailing. Clin Orthop. 1986; 212:142-54.

30. Ring D, Jupiter JB, Sanders RA, Quintero J, Santoro VM, Ganz R et al. Complex nonunion of fractures of the femoral shaft treated bay wave-plate osteosynthesis. J Bone Joint Surg Br. 1997; 79: 289-94.

31. Rosen H. Compression treatment of long bone pseudarthroses. Clin Orthop. 1979; 138:154-66.

32. Weller S, Knapp V. Treatment of noninfected pseudarthrosis of the shaft by medullary nailing. In: Chapchal G. Pseudarthrosis and their treatment. Stuttgart: Georg Thieme; 1979. p. 56-7. 